\title{
MP-MID: Multi-Protocol Oriented Middleware-level Intrusion Detection Method for Wireless Sensor Networks
}

\author{
Qi Guo ${ }^{1}$,Xiaohong $\mathrm{Li}^{1}$, Guangquan $\mathrm{Xu}^{1, *}$ and Zhiyong Feng ${ }^{1}$ \\ ${ }^{1}$ School of Computer Science and Technology \\ Tianjin University, Tianjin, China \\ *corresponding author:losin@tju.edu.cn
}

\begin{abstract}
It is very difficult to detect intrusions in wireless sensor networks (WSN), because of its dynamic network topology and diverse routing protocols. Traditional Intrusion Detection Systems (IDS) for WSN only focus attention on some one routing protocol, which lacks universality and flexibility. To solve the problem of multi-protocol intrusion detection, this paper proposes a universal method: MP-MID (Multi-Protocol Oriented Middleware-level Intrusion Detection). Our work can generate all known attack types for any routing protocol of WSN, and furthermore, all of them can be detected with the automatically generated rules. In this work, we formalize the routing protocol with the Process Algebra for Wireless Mesh Networks (AWN) language, and propose the conception of attack points to find out all attack types. Combining attack points with formalized protocol in AWN, we get co-sentences which represent the attack features in the protocol. With program slicing technology, all known attack types can be found out based on co-sentences. According to the characteristic of the key variables of the attack types, MP-MID can generate misused based detection or anomaly based detection. Our case study of ADOV (Ad hoc On-demand Distance Vector) protocol shows that our method generated all types of attacks, which outperforms other work. Experimental results show that our generated detection methods have a relatively high detection accuracy rate as we claimed. Our MP-MID method could be used as a flexible and universal tool to analyze and detect attack types for multi-protocol in WSN effectively.
\end{abstract}

Keywords: wireless sensor networks, intrusion detection, AWN, AODV 


\section{Introduction}

Wireless Sensor Networks (WSN) have become a new generation network after Internet and mobile network because of its flexibility, adaptability and reconfigurable architecture. Nowadays WSN is widely used in a variety of markets including smart homes, smart city, utility industry, etc.

There are great menaces in the WSN applications due to their highly dynamic network topologies and unpredictable environments. Intrusion detection is an essential technique in the secure protection [1]. However, most intrusion detection methods in traditional networks are unsuitable to be applied into the WSN environment directly [2], because of the limited power supply [3, 4], low transmission bandwidth [5], small memory size [1, 6] and data storage [4].

The methods of Intrusion detection Systems (IDS) can be classified into two types. One is anomaly-based detection (also called outlier detection) [7]. This is based on statistical behavior modeling. Normal operations of the members are profiled and a certain amount of deviation from the normal behavior is flagged as an anomaly. The main disadvantage of this detection type is that the normal profiles must be updated periodically, since the network behavior may change rapidly. The updating is costly to WSN. The other type is misuse-based detection (also called rule based detection)[8]. The signatures (profiles) of the previously known attacks are generated and are used as a reference to detect future attacks. The advantage of this type of detection is that it can detect known attacks accurately and efficiently; hence they have a low false positive rate. The disadvantage is that it is helpless to detect the new attack type which has not been profiled before.

Detection methods always only focus on one specific implementation of one specific routing protocol. Even if there are two or more studies of the same routing protocol, it is hard to compare the effectiveness of them. , because the same protocol maybe have different implementations. Even if the implementations are same, there is no public attack type database to support their researching. Consequently each researcher builds up his own experiment by using various attack types $[9,10]$, which prevents the comparison and extension.

To solve the problem of multi-protocol intrusion detection, this paper proposed a universal method: MP-MID (Multi-Protocol Oriented Middleware- 
level Intrusion Detection). Our work can generate all the attack types for any routing protocol of WSN, furthermore, part of which can be detected with the automatically generated rules accurately.

The remainder of this paper is arranged as follows: Section 2 introduces the related work on intrusion detection in WSN. Section 3 introduces the framework of our middleware-level intrusion detection. Section 4 improves the process Algebra for Wireless sensor Networks routing protocols (AWN). Section 5 and Section 6 analyze the attack types and their corresponding detection rules. Section 7 demonstrates an AODV case study to verify the effectiveness of our method. Section 8 concludes the paper and points out possible directions for future work.

\section{Related work}

Generally, there are two research directions for intrusion detections in WSN: frameworks and algorithms.

The first direction is about the detection frameworks. Zhang and Lee [8] proposed an agent based distributed and collaborative IDS which is compliant with the Wireless Ad Hoc Network operating conditions. As mentioned in [11], the IDS agent described in Zhang's article [12] is composed of six blocks: local data collection, local detection engine, local response, cooperative detection, global response, and secure communication. With zone based IDS [13], the network is divided into non-overlapping zones and each IDS agent broadcasts locally generated alerts inside the zone. Gateway zones are responsible for aggregation and correlation of locally generated alerts. Only gateway nodes can generate network wide alarms. Alerts indicate possible attacks and are generated by local IDS agents, while alarms indicate the final detection and can be generated only by gateway nodes.

The second direction is about the detection algorithms. Joseph [14] proposed an anomaly based cross-layer intrusion detection method. Not only did he detect the element comprehensively, but also put forward the methods to refine the data to improve the accuracy of the classification. Huang [15] proposed a method to detect the basic attack elements. He decomposed the attack scenario into different attack element combination results. Then he pointed out there are some attacks which are easy to be detected by the misuse based detection, whereas others are suitable for anomaly based detection. But in his work of misuse based detection, the monitor need to get the information of every node's route table, which costs too much for the IDS. 
Mahmood [16] took a survey of the black hole detection in AODV protocol. Hollick, Schmitt, Seipl and Steinmetz [17] adopted a 2-stage approach. First, they developed a realistic analytical model of the AODV route acquisition process. Second, they extended the work to derive a classification scheme for misbehaving nodes, including nodes of black hole behaviors [18].Dhane and Sharma [19] also aimed at detect the black hole attack in AODV. They used the misuse based detection and found three finite state machines to detect the RREP and RREQ which bring the black hole attack. But according to our methods, RERR packet also can lead to the black hole attack which haven't been considered by them.

\section{Intrusion Detection Modeling}

Fig.1 is the whole framework of this paper. The framework can generate detection methods and adjust them according to the requirement and running state. The framework consists of five parts. The top left one is environment condition, such as computation location of the collected data. These conditions are unchangeable because of the constrains of the architecture. The left bottom one is the application layer, which decides the performance requirement and security requirement with trade-off analysis. The upwards one represents the analysis of wireless sensor networks protocol, with which we can get the protocol weakness and attack types. The center one is the middleware architecture. It generates the suitable methods to detect attack types in this WSN application. The last part is the WSN nodes. They are responsible for collecting data message and detection information. Depending on the message, the Intrusion Detection System could analyze the current network state security or not.

\section{Improving AWN}

According to different requirements of applications, WSN protocol always has different implementations. For example, AODV protocol has four implementations: Kernel-AODV [20], AODV-UIUC [21], AODV-UCSB [22] and AODV-ns2 implementations. Although all of them do capture the main aspects of the AODV protocol, as specified in the AODV RFC [23], these implementations behave differently. And these differences can affect the essential features of the protocol, such as loop freedom [24] and attack analysis. 


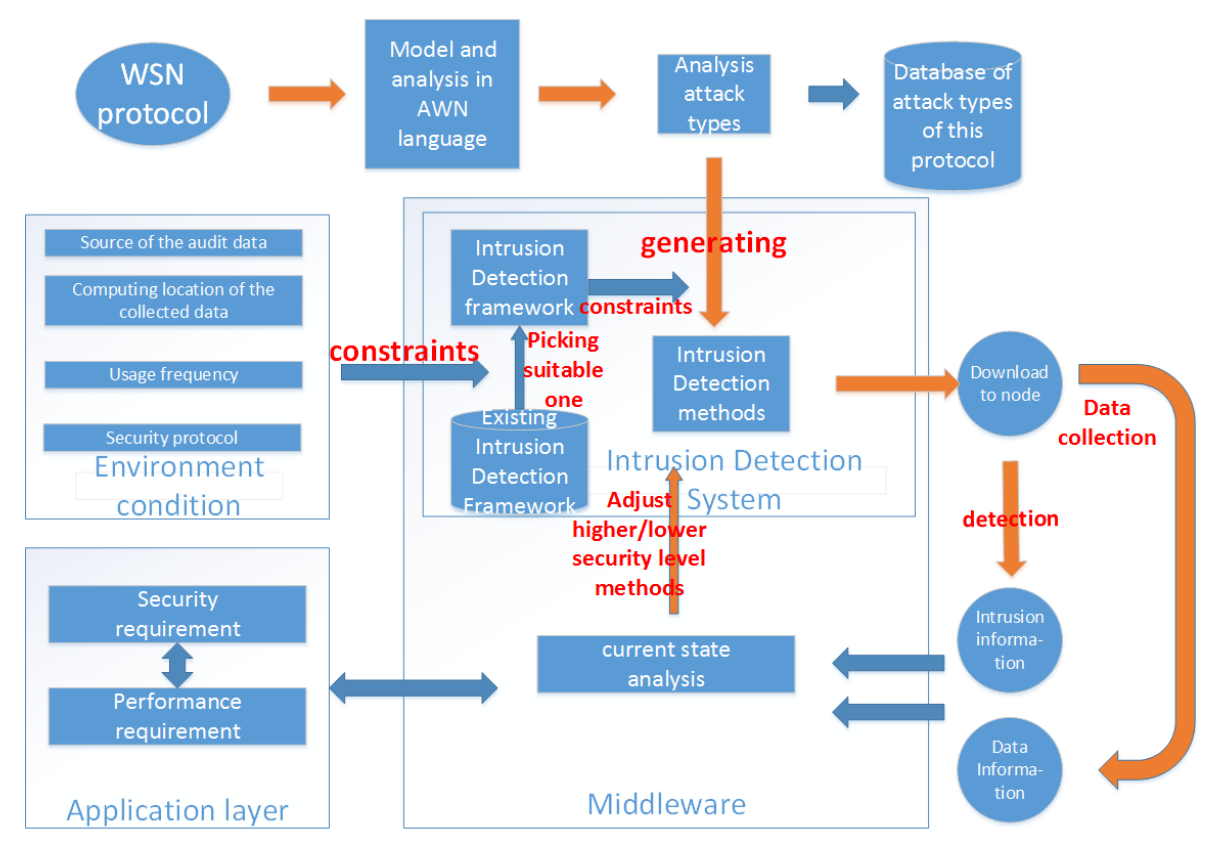

Fig. 1. Intrusion detection architecture

AWN (Algebra of Wireless Networks) [15] is a process algebra for the specification of WSN routing protocols. It allows us to embed data structures, such as route table in the protocol describing. This makes AWN more suitable to describe the routing protocol than other algebra process language, such as CSP[25] or PI-calculus[26]. In AWN, a WSN protocol is modelled as an encapsulated parallel composition of network nodes. In every node, several sequential processes running in parallel describe the protocol processing logic; between nodes, sequential processes represent the communicating with its neighbors.

We maintain the skeleton of the original AWN, and add some CSP grammars to improve the description ability. We use the "|||" symbol to describe the parallel processes without barrier. And we use "SP=event $\{$ C-like codes $\}$ " to allow the C-like languages added into the process. Furthermore, we redefine the set description grammar so that user can only use the logic operator in the set description, which improves the readability. TABLE 1 is the main primitive of the AWN language. 
Table 1. Main grammar of our improved AWN Language

\begin{tabular}{|c|c|}
\hline Expression & Description \\
\hline $\mathrm{SP}=$ event & sequential process can transform to an event \\
\hline $\mathrm{SP}=\mathrm{X}(\exp 1, \exp 2, \ldots)$ & process name $\mathrm{X}$ with arguments \\
\hline $\mathrm{SP}=\mathrm{SP} 1 \mid \| \mathrm{SP} 2$ & $\begin{array}{c}\text { SP2 } \text { and } \mathrm{SP} 1 \text { run concurrently } \\
\text { without barrier synchronization }\end{array}$ \\
\hline event=event1 $\rightarrow$ event 2 & event1 acts after event2 \\
event $=[\varphi]$ event & $\begin{array}{c}\varphi \text { is a data formula, } \\
\text { wait until } \varphi \text { evaluates to true, } \\
\text { act as event followed }\end{array}$ \\
\hline $\begin{array}{c}\text { event= }=\{\mathrm{C}-\text { like code } \\
\text { or set-language }\}\end{array}$ & $\begin{array}{c}\text { An event can be embedded with C code } \\
\text { or set description language }\end{array}$ \\
\hline event=SP & event call the sequential process \\
\hline
\end{tabular}

\section{Generating Attack Types}

To generate all known attack types of the formalized protocol, we should make sure that every attacker's characters are taken into consideration. As shown in Fig.2, we combine the universal characters of the attack with the formalized protocol and then search out all the potential attack types (according to these characters).

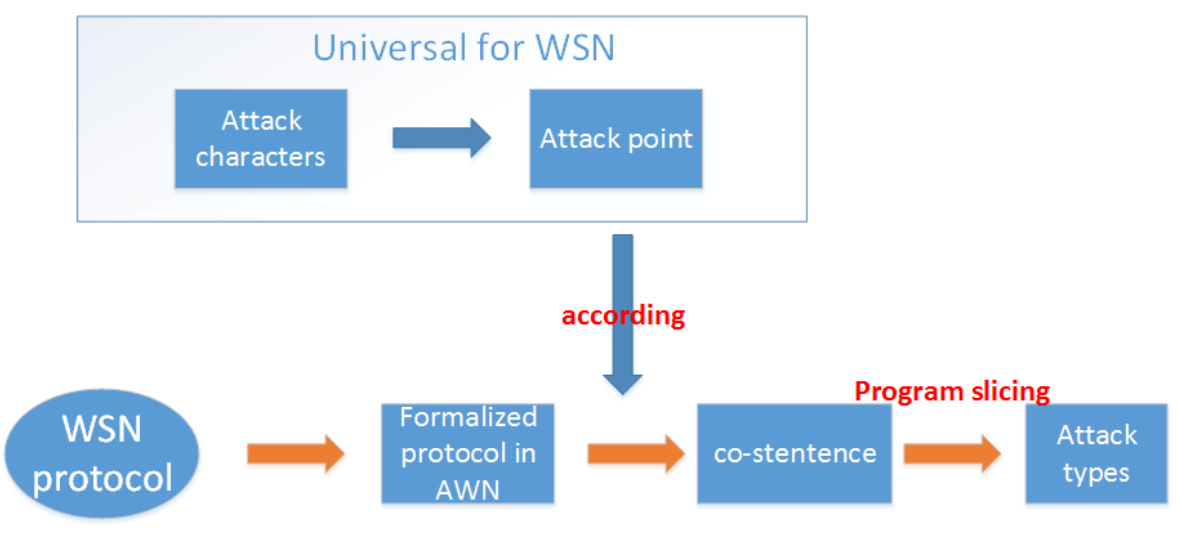

Fig. 2. process of generation attack types 
Table 2. Relationship between attack point and attack categories

\begin{tabular}{|c|c|c|c|}
\hline $\begin{array}{c}\text { Ismail's } \\
\text { categories }\end{array}$ & $\begin{array}{l}\text { attack } \\
\text { points }\end{array}$ & $\begin{array}{l}\text { Ismail's } \\
\text { categories }\end{array}$ & $\begin{array}{l}\text { attack } \\
\text { points }\end{array}$ \\
\hline \multirow{2}{*}{$\begin{array}{l}\text { Attempted } \\
\text { break-in }\end{array}$} & \multirow[t]{2}{*}{ penetrating } & Sybil & penetrating \\
\hline & & Cloning & penetrating \\
\hline Masquerade & penetrating & Wormhole & penetrating \\
\hline Penetration & penetrating & Blackhole & penetrating \\
\hline \multirow{4}{*}{ DoS } & \multirow{4}{*}{$\begin{array}{c}\text { penetrating } \\
\text { preventing } \\
\text { breaking } \\
\text { consuming }\end{array}$} & Hello Flood & consuming \\
\hline & & \multirow{2}{*}{$\begin{array}{l}\text { Acknowledgement } \\
\text { Spoofing }\end{array}$} & breaking \\
\hline & & & penetrating \\
\hline & & \multirow[t]{2}{*}{ Manipulating } & breaking \\
\hline \multirow{2}{*}{$\begin{array}{l}\text { Malicious } \\
\text { use }\end{array}$} & \multirow{2}{*}{$\begin{array}{l}\text { penetrating } \\
\text { consuming }\end{array}$} & & penetrating \\
\hline & & Selective Forwarding & penetrating \\
\hline
\end{tabular}

\subsection{Attack points}

There are infinitely many attack types in the WSNs applications. To analyze all of them, classification and characterization is necessary.According to the ability and purpose of the attacker, Ismail Butun [27] gave a kind of partition of the attack types: Attempted break-in, Masquerade, Penetration, DoS and Malicious use. According to the process and scene of the attacker, Hemanta [28] identify and categorize attack types as follow: Denial of Service, Sybil, Wormhole, Sinkhole(black hole), Manipulating Routing Information, Selective Forwarding, Hello Flood, Acknowledgement Spoofing, Cloning.

This paper proposed a new kind of partition of attack types according to attacker behaviors, thus four attack types are gotten.

breaking: attacker breaks original route to make some nodes unusable

preventing: attacker prevents new route establishing to make network unusable

penetrating: attacker puts itself into others' route pathes

consuming: attacker consumes others' resource, especial for energy, CPU and bandwidth

Definition 1:attack point is the above four types of attacker behaviors.

To our best knowledge, these attack points can characterise all the attack types. Table 2 shows the relationship between attack point and attack types. 


\subsection{Attack point in the AWN language}

Not all the nodes which act as the attack point behaviors are attackers. But all the attackers must act as one of the attack points. When a normal node $\mathrm{A}$ find a legal route path to $\mathrm{D}$ via node $\mathrm{B}$, node $\mathrm{A}$ will add $\mathrm{B}$ into its route path to $\mathrm{D}$. But, in the black hole attack, a malicious node $\mathrm{M}$, will as well try to make all the nodes $A_{1}, A_{2}, . ., A_{n}$ add $\mathrm{M}$ into their route path to $\mathrm{D}$ (maybe sink node), by sending malicious message to $A_{1}, A_{2}, . ., A_{n}$. This paper firstly detects all the process behaviors which trigger attack points, and filters the non-attack ones in the second step.

While a normal node A executing the protocol, the only method to attack $\mathrm{A}$ is that sending malicious message to A. And during the attacking, A must trigger at least one of the attack points, according to the analysis above.

Firstly, we focus on the attack points. Some AWN sentences in the protocol are picked up, and mark with execution conditions. When the process run this sentences and satisfy its execution condition, the attack point will be triggered and the atomic attack purpose will be achieved.

Definition 2: co-sentence is the corresponding sentence of the attack points.

There are the four rules to pick up the co-sentences.

Rule 1: breaking. Find out all the deleting route sentences and marking route invalid sentences. The condition set as "many times".

Rule 2: preventing. Find out all the sentences updating route table. The condition set as "never execution"

Rule 3: penetrating. Find out all the sentences unicasting data packets. The condition set as "destination $==$ MID".

Rule 4: consuming. Find out all the broadcast and groupcast sentences. The condition set as "many times".

\subsection{Program Slicing}

Program slicing [29] is a decomposition technique that extracts from a program those statements relevant to a particular computation. We use this tech with a few addition.

Tradition program slicing only focus on the variable $v$ and statement $s$. In this paper, we add the condition into the focuses. A co-sentence can be described as a triple $(s, v, c)$, where $s$ represents one statement of the protocol in AWN language, $v$ represents the key variables which can effect the attack consequence and $c$ represents the condition when process executes 
Table 3. Rules of the condition transform

\begin{tabular}{|c|c|}
\hline $\begin{array}{c}\text { sentence type in } \\
\text { program slicing }\end{array}$ & condition transform \\
\hline $\mathrm{a} \leftarrow \mathrm{b}$ & replace the variable a to b \\
\hline $\mathrm{a} \leftarrow$ RouteTable.get(key) & $\begin{array}{c}\text { replace the variable a to } \\
\text { ANY entry of the routetable }\end{array}$ \\
\hline if $\left(\right.$ cond $\left._{1}\right)$ & add the cond $d_{1}$ to condition \\
\hline$\left[\right.$ cond $\left._{2}\right]$ event & add the cond $d_{2}$ to condition \\
\hline
\end{tabular}

the statement.

For every co-sentence triple(s,v,c), we list out all the correlation sentences according to the backward program slicing of $(\mathrm{s}, \mathrm{v})$ as initial point. The table3 is the operation to the condition.

When the statement arrive at the receiving message sentence, then we mark that, there is a potential attack type. It means that if the attacker could set elaborate value to the key variable in the message and make the condition true, then send this malicious message to the normal node. The normal node will be affected and act as the attack point behaviors. From the algorithm above, we can generate all the potential attack types according to the attack point.

\section{Intrusion Detection Methods}

According to key variables, this paper generate two detection methods: misuse base detection and anomaly based detection.

Misuse based detection is simple to install and efficient to operate.On the other hand, they can only detect the specific attack types.

Anomaly based detection can detect a large amount of attack types and their variants. Unfortunately they have high computational complexity and high energy consumption requiring large amounts of data samples. Besides, they also need efficient analytic tools to analyze large amount of audit data and a mass memory space to store them.

The ideal method is misuse based detection because of its efficiency and accuracy. We focus on the key variables in the suspicious messages. If all variables do not rely on local variables. We can generate a detection rule to this variable. Fig. 3 shows an example of this type of detection rules. 


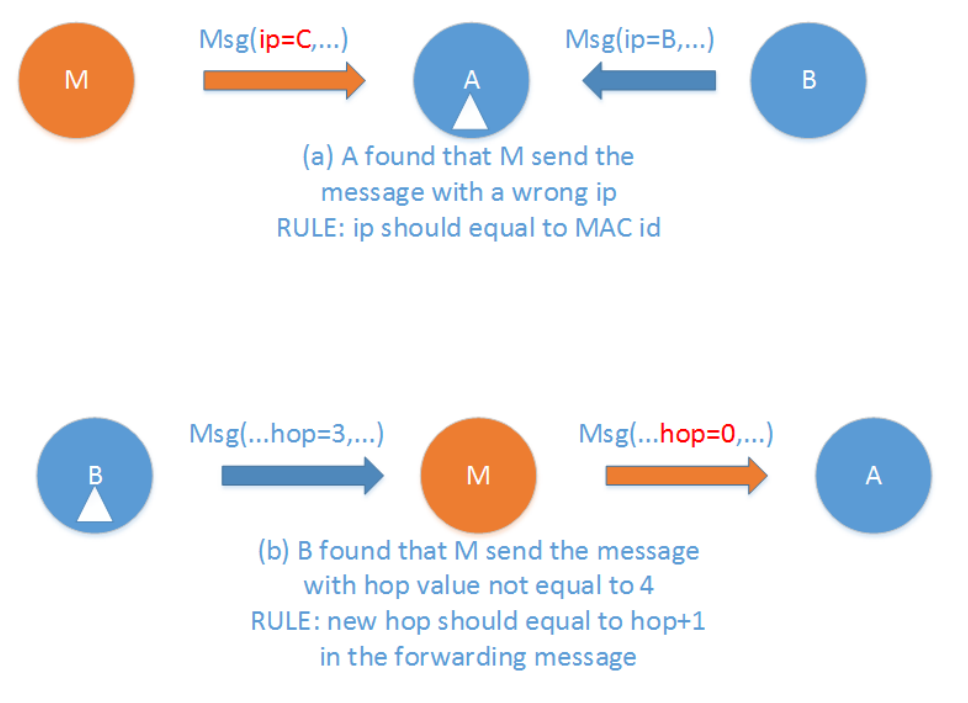

Fig. 3. Misuse detection method

If the key variables are relevant with local variables, such as route table or buffer, this paper use an anomaly based detection method. All the attack types use the malicious message to attack normal node. The regular patten of the sending message should be checked. Taking the whole protocol process into consider, the more messages one node receiving, the more messages this node maybe send. We build the detection methods as follow:

If the protocol describe $\mathrm{n}$ types of the message $T_{1}, T_{2}, \ldots, T_{n}$. And marking that node A have received Receive $_{T_{i}}$ message of type $T_{i}$ in a given period. Similar, we mark $\operatorname{Send}_{T_{i}}$ for number of sent messages. We detect the ratios of these number.

$$
\begin{aligned}
& \frac{\text { Receive }_{T_{i}}}{\operatorname{send}_{T_{i}}} \quad(1 \leq i \leq n) \\
& \frac{\operatorname{Receive}_{T_{i}}}{\operatorname{send}_{T_{j}}} \quad(1 \leq i<j \leq n)
\end{aligned}
$$

After detecting and comparing the ratios, the attacker will be found especial for those flooding and black hole attack types. 


\section{Case study}

In this section, we will use AODV protocol analysis as an example to show our method.

\subsection{AODV protocol}

Ad hoc on-demand distance vector (AODV)[30] protocol was developed by Perkins and Royer. It belongs to Source-initiated routing protocol and it is widely used in many Wireless Sensor Networks applications. This paper will analyze the most robust implementation of AODV according to [6].

\subsection{Formalizing in $A W N$}

In AODV protocol, the main process consist of three sub-processes: Sending message, Receiving message and Handling Message. In Sending process, it firstly check the sending buffer, if the buffer not empty, then do the sending operation. After unicasting a message, the main thread call back the Sending process and it will create another thread waiting for an ACK back to notify whether receiver had gotten the message. In Receiving process, it firstly execute an event(recv) which parallel with the channel event(recv). After receiving from channel, it write the receive buffer and left the Handling process to cope. The Handling process reading from receiving buffer, deal with different message types. Fig.4 shows part of the AODV in AWN language.

\subsection{Attack points and attack types}

According to the methods in Section 4, all attack types are listed in Table.4. In security term, it is impossible to find out all the attackers in AODV. Maybe in future, there will emerge some unknown attackers and attack types. But up to now, all the known attack types of AODV are listed. Comparing to other researches on intrusion detection of AODV protocol [31, $18,32,15]$, we find out the most attack types than any other works.

\subsection{Detection metheds and experiment results}

According to the method in Section5, we get the detection methods, listed in the Table.5. The $*$ means this attack type should use anomaly based detection methods. 
AODV () = HandleMsg() || SendMsg() || RecMsg()

HandleMgg( ) = ['recBuffer.igEmpty ()]

doHandle 1

mgg = recBuffer.pop()

if (mag.type - newpktType)

newpkt (msg.da , msg.dip):

elseif (msg.type - pktType)

pkt (msq.da, msg.dip, msg.0ip)

elseif (mgg.type - rreqType)

rtUpdate (msg. sip, O, unk, val, 1, msg.s

rreq (msg.hops , msg.rreqid , msg.dip , 1

)

elseif (mgg.type - rrepType)

rtUpdate (msg.sip, 0, unk, val, 1, msg.sip, nul1)

rrep (msg.hops , msg.dip， msg.dsn，msg.oip，mgg.sip) :

elseif (mgg.type - rerrType)

rtUpdate (msg.sip, 0, unk, val, $1, x 60$

rerr (mgg.dests, mgg.sip);

endif

SendMsg ()$=[$ 'sendBuffer.1sEmpty ()$]$

dosend

ste $=$ sendBuffer top ()

rte=get $(r t$, ste. dip) :

if (rte.valid) i

unicast (rte.nextHop, ste.pktTy

SendMgg ()

[ack (ste.sn)]

handleAck 1

if (ack=suc) (

)

sendBuffer.drop (s

if (ack!=suc) !

Fig. 4. Part of AODV protocol in AWN language

Table 4. Attack types of AODV

\begin{tabular}{|c|c|c|}
\hline Statement & Execution Condition & Attack Type \\
\hline $\begin{array}{c}\text { line 11: } \\
\text { rtUpdate(.,.,.,...,msg.sip,..) }\end{array}$ & $\begin{array}{c}\text { msg.sip } \leftarrow \text { MID } \\
\text { msg.type }==\text { rreq }\end{array}$ & attack type 1 \\
\hline $\begin{array}{c}\text { line 16: } \\
\text { rtUpdate(.,.,.,...,msg.sip,..) }\end{array}$ & $\begin{array}{c}\text { msg.sip } \leftarrow \text { MID } \\
\text { msg.type }==\text { rrep }\end{array}$ & attack type 2 \\
\hline $\begin{array}{c}\text { line 21: } \\
\operatorname{rtUpdate}(., ., \ldots, . ., \mathrm{msg} . \operatorname{sip}, .)\end{array}$ & $\begin{array}{c}\text { msg.sip } \leftarrow \text { MID } \\
\text { msg.type }==\text { rerr }\end{array}$ & attack type 3 \\
\hline $\begin{array}{c}\text { line } 12: \\
\operatorname{rreq}(\mathrm{msg} . h o p s, ., \text { dip }, ., ., \mathrm{msg} . \operatorname{sip}, .)\end{array}$ & $\begin{array}{c}\text { msg.sip } \leftarrow \text { MID } \\
\text { msg.hops }<\text { rt.get }(\text { dip }) . h o p s\end{array}$ & attack type 4 \\
\hline $\begin{array}{c}\text { line } 12: \\
\operatorname{rreq}(., ., \text { dip,msg.dsn,..,msg.sip,..) }\end{array}$ & $\begin{array}{c}\text { msg.sip } \leftarrow \text { MID } \\
\text { msg.dsn }>\text { rt.get }(\text { dip }) . d s n\end{array}$ & attack type 5 \\
\hline $\begin{array}{c}\text { line } 17: \\
\operatorname{rrep}(\text { msg.hops, ...,oip,msg.sip) }\end{array}$ & $\begin{array}{c}\text { msg.sip } \leftarrow \text { MID } \\
\text { msg.hops }<\text { rt.get (oip).hops }\end{array}$ & attack type 6 \\
\hline $\begin{array}{c}\text { line } 22: \\
\operatorname{rrer}(\text { msg.dests,msg.sip) }\end{array}$ & $\begin{array}{c}\text { msg.sip } \leftarrow \text { MID } \\
\text { EXECUTE MANY TIMES }\end{array}$ & attack type 7 \\
\hline $\begin{array}{c}\text { line 35: } \\
\text { handleAck }\end{array}$ & $\begin{array}{c}\text { ack! }=\text { suc } \\
\text { EXECUTE MANY TIMES }\end{array}$ & attack type 8 \\
\hline $\begin{array}{c}\text { line } 12: \\
\operatorname{rreq}(., ., ., ., ., . . .)\end{array}$ & EXECUTE MANY TIMES & attack type 9 \\
\hline
\end{tabular}


Table 5. Detection metheds of AODV

\begin{tabular}{|c|c|}
\hline Attack Type & Detection methods \\
\hline attack type 1 & $\begin{array}{c}\text { if msg.type }==\text { rreq then check } \\
\text { msg.sip }==\text { MAC.ip }\end{array}$ \\
\hline attack type 2 & $\begin{array}{c}\text { if msg.type }==\text { rrep then check } \\
\text { msg.sip }==\text { MAC.ip }\end{array}$ \\
\hline attack type 3 & $\begin{aligned} \text { if msg.type } & ==\text { rerr then check } \\
\text { msg.sip } & ==\text { MAC.ip }\end{aligned}$ \\
\hline attack type 4 & $\begin{array}{c}\text { if msg.type }==\text { rreq then check } \\
\text { msg.hops }==\text { msgrecv.hops }+1 \\
\text { msg and msgrecv has same rreqid }\end{array}$ \\
\hline attack type 5 & $\begin{array}{c}\text { if msg.type }==\text { rreq then check } \\
\text { msg.dsb==msgrecv.dsn } \\
\text { msg and msgrecv has same rreqid }\end{array}$ \\
\hline attack type 6) & $\begin{array}{c}\text { if msg.type }==\text { rrep then check } \\
\text { msg.hops }==\text { msgrecv.hops }+1 \\
*\end{array}$ \\
\hline attack type 7 & $\begin{aligned} \text { if msg.type }= & =\text { rerr then check } \\
\text { msg.sip } & ==\text { MAC.ip } \\
& *\end{aligned}$ \\
\hline attack type 8 & $*$ \\
\hline attack type 9 & $*$ \\
\hline
\end{tabular}

\subsection{1. experiment environments}

We used the network simulator (ns-2). A hypothetical network was constructed for the simulation purpose and then monitored for a number of parameters. We simulate our model from 10 to 50 nodes. Each node in the AODV protocol is assigned an initial position without movement. And the nodes join or left the network at a random time. The packets include route messages and data messages. The detection distribute on every node and set as wiretapping mode to sniffer each neighbors.

\subsection{2. experiment results}

In the first experiment, we simulate only one attacker in the networks. It acts each type of attack 100 times, then check the detection accuracy of these detection methods. In the next experiment, we add the attacker to $10 \%$ percents of the whole nodes, and check the accuracy of the detection. 

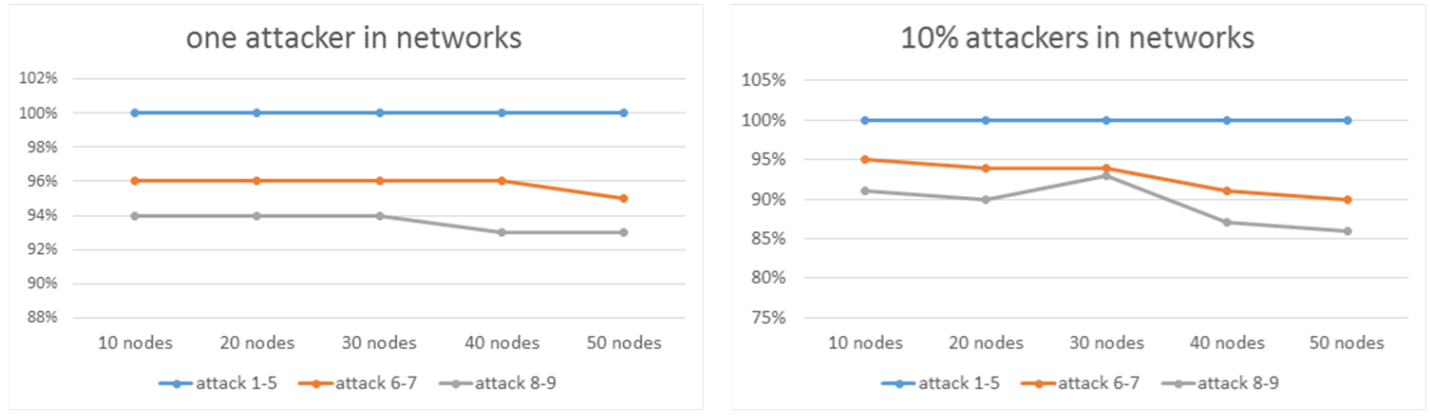

Fig. 5. Result of experiments

The results in Fig. 5 shows that, the rule based detection are perfect to detect the corresponding attacks. But the mix of rule based detection and anomaly base detection are less accuracy than it. And at last, the pure anomaly based detection has the least accuracy. Besides, with the growth of the network scale, the accuracy decrease down gradually. And with the attacker become more, the networks becomes unstable. Thus the detection accuracy will drop down.

\section{CONCLUSION AND FUTURE WORK}

To solve the problem of multi-protocol intrusion detection, we propose a MP-MID method to generate attack types from any WSN routing protocol. We formalize the protocol and analyze the attack points. From those, we can generate all the Attack Types. Attack Types can be used in generating experiment environment for intrusion detection and protocol analysis. Further, the intrusion detection methods are generated, based on which we can build a concrete Intrusion Detection System. Our MP-MID method outperforms other work in that it could be used as a flexible and universal tool to analyze and detect attack types for multi-protocol of WSN effectively.

In further work, we will improve the IDS to detect attacks by introducing application layer information. To improve the usability of our method, we should realize the transformation between C-like languages (such as nesc and ns-2 language) and the AWN. 


\section{ACKNOWLEDGMENT}

This work has partially been sponsored by the National Science Foundation of China (No. 91118003, 61272106, 61572355, 61572349) and 985 funds of Tianjin University, Tianjin Research Program of Application Foundation and Advanced Technology under grant No. 15JCYBJC15700 and No. 14JCTPJC00517.

\section{Reference}

[1] K. Gai, M. Qiu, L. Tao, Y. Zhu, Intrusion detection techniques for mobile cloud computing in heterogeneous $5 \mathrm{~g}$, Security and Communication Networks.

[2] I. Butun, R. Sankar, A brief survey of access control in wireless sensor networks, in: Consumer communications and networking conference (CCNC), 2011, pp. 1118-1119.

[3] M. Qiu, W. Gao, M. Chen, J.-W. Niu, L. Zhang, Energy efficient security algorithm for power grid wide area monitoring system, Smart Grid, IEEE Transactions on 2 (4) (2011) 715-723.

[4] M. Qiu, L. Zhang, Z. Ming, Z. Chen, X. Qin, L. T. Yang, Securityaware optimization for ubiquitous computing systems with seat graph approach, Journal of Computer and System Sciences 79 (5) (2013) 518529.

[5] H. Dai, S. Zhao, J. Zhang, M. Qiu, L. Tao, Security enhancement of cloud servers with a redundancy-based fault-tolerant cache structure, Future Generation Computer Systems.

[6] A. Fehnker, R. van Glabbeek, P. Höfner, A. McIver, M. Portmann, W. L. Tan, A process algebra for wireless mesh networks, in: Programming Languages and Systems, Springer, 2012, pp. 295-315.

[7] A. Saini, K. K. Sharma, S. Dalal, A survey on outlier detection in wsn, International Journal of Research Aspects of Engineering and Management 1 (2) (2014) 69-72.

[8] Y. Zhang, W. Lee, Y.-A. Huang, Intrusion detection techniques for mobile wireless networks, Wireless Networks 9 (5) (2003) 545-556. 
[9] R. Mitchell, R. Chen, A survey of intrusion detection in wireless network applications, Computer Communications 42 (2014) 1-23.

[10] A. R. Sardar, R. R. Sahoo, M. Singh, S. Sarkar, J. K. Singh, K. Majumder, Intelligent intrusion detection system in wireless sensor network, in: Proceedings of the 3rd International Conference on Frontiers of Intelligent Computing: Theory and Applications (FICTA) 2014, Springer, 2015, pp. 707-712.

[11] T. Anantvalee, J. Wu, A survey on intrusion detection in mobile ad hoc networks, in: Wireless Network Security, Springer, 2007, pp. 159-180.

[12] Y. Zhang, W. Lee, Intrusion detection in wireless ad-hoc networks, in: Proceedings of the 6th annual international conference on Mobile computing and networking, ACM, 2000, pp. 275-283.

[13] B. Sun, K. Wu, U. W. Pooch, Zone-based intrusion detection for mobile ad hoc networks, Int. Journal of Ad Hoc and Sensor Wireless Networks $2(3)$.

[14] J. F. C. Joseph, B.-S. Lee, A. Das, B.-C. Seet, Cross-layer detection of sinking behavior in wireless ad hoc networks using svm and fda, Dependable and Secure Computing, IEEE Transactions on 8 (2) (2011) $233-245$.

[15] Y.-a. Huang, W. Lee, Attack analysis and detection for ad hoc routing protocols, in: Recent advances in intrusion detection, Springer, 2004, pp. 125-145.

[16] R. Mahmood, A. Khan, A survey on detecting black hole attack in aodvbased mobile ad hoc networks, in: High Capacity Optical Networks and Enabling Technologies, 2007. HONET 2007. International Symposium on, IEEE, 2007, pp. 1-6.

[17] M. Hollick, J. B. Schmitt, C. Seipl, R. Steinmetz, The ad hoc on-demand distance vector protocol: an analytical model of the route acquisition process, in: Wired/Wireless Internet Communications, Springer, 2004, pp. 201-212.

[18] X. Wang, T.-l. Lin, J. S. Wong, Feature selection in intrusion detection system over mobile ad-hoc network. 
[19] A. Dhane, S. Sharma, Modeling and analysis of sequence number attack and its detection in aodv, in: Networks, 2008. ICON 2008. 16th IEEE International Conference on, IEEE, 2008, pp. 1-6.

[20] L. Klein-Berndt, Kernel aodv, National Institute of Standards and Technology, Technology Administration, US Department of Commerce 66.

[21] V. Kawadia, Y. Zhang, B. Gupta, System services for ad-hoc routing: Architecture, implementation and experiences, in: Proceedings of the 1st international conference on Mobile systems, applications and services, ACM, 2003, pp. 99-112.

[22] I. D. Chakeres, E. M. Belding-Royer, Aodv routing protocol implementation design, in: Distributed Computing Systems Workshops, 2004. Proceedings. 24th International Conference on, IEEE, 2004, pp. 698703.

[23] C. Perkins, E. Belding-Royer, S. Das, Ad hoc on-demand distance vector (aodv) routing, Tech. rep. (2003).

[24] R. Van Glabbeek, P. Höfner, W. L. Tan, M. Portmann, Sequence numbers do not guarantee loop freedom: Aodv can yield routing loops, in: Proceedings of the 16th ACM international conference on Modeling, analysis \& simulation of wireless and mobile systems, ACM, 2013, pp. 91-100.

[25] C. A. R. Hoare, Communicating sequential processes, Communications of the ACM 21 (8) (1978) 666-677.

[26] R. Milner, Communicating and mobile systems: the pi calculus, Cambridge university press, 1999.

[27] I. Butun, S. D. Morgera, R. Sankar, A survey of intrusion detection systems in wireless sensor networks, Communications Surveys \& Tutorials, IEEE 16 (1) (2014) 266-282.

[28] H. K. Kalita, A. Kar, Wireless sensor network security analysis, International Journal of Next-Generation Networks (IJNGN) 1 (1) (2009) $1-10$. 
[29] M. Ward, H. Zedan, Slicing as a program transformation, ACM Transactions on Programming Languages and Systems (TOPLAS) 29 (2) (2007) 7 .

[30] C. Perkins, E. Belding-Royer, S. Das, Ad hoc on-demand distance vector (aodv) routing, Tech. rep. (2003).

[31] S. Kurosawa, H. Nakayama, N. Kato, A. Jamalipour, Y. Nemoto, Detecting blackhole attack on aodv-based mobile ad hoc networks by dynamic learning method., IJ Network Security 5 (3) (2007) 338-346.

[32] B. Sun, Y. Guan, J. Chen, U. W. Pooch, Detecting black-hole attack in mobile ad hoc networks. 